\title{
FREE TOPOLOGICAL GROUPS AND DIMENSION $\left({ }^{1}\right)$
}

\author{
BY \\ CHARLES JOINER
}

ABSTRACT. For a completely regular space $X$ we denote by $F(X)$ and $A(X)$ the free topological group of $X$ and the free Abelian topological group of $X$, respectively, in the sense of Markov and Graev.

Let $X$ and $Y$ be locally compact metric spaces with either $A(X)$ topologically isomorphic to $A(Y)$ or $F(X)$ topologically isomorphic to $F(Y)$. We show that in either case $X$ and $Y$ have the same weak inductive dimension. To prove these results we use a Fundamental Lemma which deals with the structure of the topology of $F(X)$ and $A(X)$. We give other results on the topology of $F(X)$ and $A(X)$ and on the position of $X$ in $F(X)$ and $A(X)$.

1. Introduction. The purpose of this paper is to continue the study of free topological groups begun by Markov [10] and Graev [6]. Graev showed that if $X$ and $Y$ are compact metric spaces such that $A(X)$ and $A(Y)$ are topologically isomorphic, then $X$ and $Y$ have the same dimension. In Theorem 2 we strengthen this result by showing that if $X$ and $Y$ are locally compact metric spaces such that $A(X)$ and $A(Y)$ are topologically isomorphic, then $X$ and $Y$ have the same weak inductive dimension. In Theorem 3 we give the analogous result for $F(X)$.

In order to prove these results on dimension we need a convenient way to study the topology which the free topology on $F(X)$ induces on $Y$. Let $F_{n}(X)$ be the set of all elements of $F(X)$ with length at most $n$. We define $A_{n}(X)$ similarly. We prove a Fundamental Lemma which says that if $x_{1}^{\epsilon_{1}} x_{2}^{\epsilon_{2}} \cdots x_{n}^{\epsilon_{n}}$ is the reduced representation of a point of $F(X)$ where $x_{i} \in X$ and $\epsilon_{i}$ is 1 or -1 for $i=1,2, \ldots, n$, then a fundamental system of neighborhoods of $x_{1}^{\epsilon_{1}} x_{2}^{\epsilon} \ldots$ $x_{n}^{\epsilon_{n}}$ in $F_{n}(X)$ is formed by the family of all sets of the form $U_{1}^{\epsilon_{1}} U_{2}^{\epsilon_{2}} \cdots U_{n}^{\epsilon_{n}}$ where $U_{i}$ is a neighborhood of $x_{i}$ in $X$ for $i=1,2, \ldots, n$. The analogous result is true for $A(X)$.

In addition to our Fundamental Lemma we need Theorem 1 in order to

Received by the editors November 7, 1972 and, in revised form, June 23, 1975.

AMS (MOS) subject classifications (1970). Primary 22 A05, 54F45; Secondary 54D20.

Key words and phrases. Free topological group, free Abelian topological group, dimension, compactness, paracompactness, automorphism, varieties of topological groups.

(1) This work was supported in part by National Science Foundation Grants GP-8394 and GP-20632. The author wishes to thank Professor Ky Fan for his encouragement and guidance. 
prove Theorems 2 and 3. Theorem 1 says that if $X$ is any completely regular space and $Y$ is a compact subset of $A(X)$ then $Y \subset A_{k}(X)$ for some positive integer $k$.

Another consequence of Theorem 1 is Theorem 4 which says that if $X$ is not discrete then $F(X)$ and $A(X)$ are not locally compact. We also give an example of a paracompact space $X$ such that $F(X)$ and $A(X)$ are not normal.

Finally let $\varphi$ be a topological isomorphism of $F(X)$ onto itself. Then in Theorem 5 we show that $\varphi(X)$ algebraically generates $F(X)$ as its free topological group. An analogous result holds for $A(X)$.

2. Preliminaries. Before going into the main results of this paper we need some background material. Let $X$ be a completely regular space. In this paper we use the definition of the free topological group $F(X)$ of $X$ given by Graev [6]. We also adopt the notation used by Graev. Unless otherwise stated, all topological spaces and topological groups are Hausdorff and $X$ will denote a completely regular space.

Let $e$ be any point of $X$. The free topological group $F(X)$ of the space $X$ is defined to be the unique topological group $F(X)$ which satisfies the following three properties.

(1) $F(X)$ contains $X$ as a subspace.

(2) There is no proper closed subgroup of $F(X)$ which contains $X$.

(3) If $\varphi$ is a continuous mapping of $X$ into a topological group $G$ for which $\varphi(e)$ is the identity of $G$, then $\varphi$ can be extended to a continuous homomorphism $\Phi$ of the topological group $F(X)$ into $G$.

The definition of the free Abelian topological group $A(X)$ of the space $X$ may be obtained by keeping (1) and (2) as they are and by considering only Abelian topological groups $G$ in (3). The existence and uniqueness of $F(X)$ and $A(X)$ follow from Graev.

It also follows that $X$ algebraically generates $F(X)$ and $A(X)$. From this we conclude that (2) may be replaced in the definitions of $F(X)$ and $A(X)$ by

(2') $X$ generates $F(X)$ algebraically.

In fact, Graev showed that the free topological group of $X$ may be obtained by taking $X \backslash\{e\}$ as a set of generators for a free algebraic group $G$. Then we identify the point $e$ of the space $X$ with the identity element of the group $G$. If $G$ is given the strongest topology which makes it into a topological group and also induces the original topology on $X$, then $G$ becomes the free topological group of $X$. Similar remarks can be made about free Abelian topological groups.

We call the topology on $F(X)$ which makes $F(X)$ into a free topological group the free topology on $F(X)$ The free topology on $A(X)$ is defined similarly. By the length of an element $\bar{x} \in F(X)$ we mean the smallest integer $n$ such 
that $\bar{x}=x_{1}^{\epsilon_{1}} x_{2}^{\epsilon_{2}} \cdots x_{n}^{\epsilon_{n}}$ where $x_{i} \in X$ and $\epsilon_{i}$ is 1 or -1 for $i=1,2, \ldots, n$. The length of the word $y_{1}^{\epsilon_{1}} y_{2}^{\epsilon_{2}} \cdots y_{k}^{\epsilon_{k}}$, where $y_{i} \in X$ and $\epsilon_{i}$ is 1 or -1 for $i=$ $1,2, \ldots, k$, is $k$, even if the word can be reduced to something shorter.

Throughout this paper, unless otherwise stated, $e$ will denote the identity of $F(X)$, and 0 the identity of $A(X)$. Unless otherwise stated, symbols like $x_{i}$ and $y_{i}$ will denote points in $F_{1}(X)$ or $A_{1}(X)$.

Suppose $X$ is a metric space. Graev gave a procedure for extending a metric on $X$ to a metric on $F(X)$ which is compatible with the group structure of $F(X)$ and whose topology is weaker than the free topology for $F(X)$. Suppose $X$ is a completely regular space. Then the procedure will also extend a pseudo-metric on $X$ to $F(X)$. If the pseudo-metric topology on $X$ is weaker than the given topology on $X$, then the topology induced on $F(X)$ by the extended pseudo-metric is weaker than the free topology on $F(X)$. Similar remarks can be made for the group $A(X)$.

As the above mentioned procedure is basic to this paper we outline it for the reader. Let $\rho$ be a pseudo-metric on the space $X$. For convenience we will denote the extension by $\rho$ also. We first extend $\rho$ to $F_{1}(X)$ by $\rho\left(x^{-1}, y^{-1}\right)=$ $\rho(x, y)$ and $\rho\left(x, y^{-1}\right)=\rho\left(x^{-1}, y\right)=\rho(x, e)+\rho(e, y)$ for any points $x$ and $y$ in $X$.

Next we extend $\rho$ to all of $F(X)$. Suppose $\bar{x}$ and $\bar{y}$ are two elements of $F(X)$. We define

$$
\rho(\bar{x}, \bar{y})=\inf \left\{\sum_{i=1}^{k} \rho\left(x_{i}^{\prime}, y_{i}^{\prime}\right): x_{1}^{\prime} x_{2}^{\prime} \cdots x_{k}^{\prime}=\bar{x} \text { and } y_{1}^{\prime} y_{2}^{\prime} \cdots y_{k}^{\prime}=\bar{y}\right\} .
$$

The above infimum is taken over all representations $x_{1}^{\prime} x_{2}^{\prime} \cdots x_{k}^{\prime}$ of $\bar{x}$ and $y_{1}^{\prime} y_{2}^{\prime}$ $\cdots y_{k}^{\prime}$ of $\bar{y}$ which have the same length. The points $x_{i}^{\prime}$ and $y_{i}^{\prime}$ are in $F_{1}(X)$ for $i=1,2, \ldots, k$. We allow $k$ to vary.

Graev showed that the distance $\rho(\bar{x}, \bar{y})$ is actually achieved for some representations of $\bar{x}$ and $\bar{y}$. That is, $\rho(\bar{x}, \bar{y})=\Sigma_{i=1}^{k} \rho\left(x_{i}^{\prime}, y_{i}^{\prime}\right)$ for some particular representations $x_{1}^{\prime} x_{2}^{\prime} \cdots x_{k}^{\prime}$ of $\bar{x}$ and $y_{1}^{\prime} y_{2}^{\prime} \cdots y_{k}^{\prime}$ of $\bar{y}$.

Let $\left\{\rho_{\nu}\right\}_{\nu \in I}$ be the family of all continuous pseudo-metrics on a completely regular space $X$. Extend $\rho_{\nu}$ to $F(X)$ for every $\nu \in I$. Let $T$ be the least upper bound of the topologies on $F(X)$ determined by the $\rho_{\nu}$ for $\nu \in I$. Then $T$ induces the original topology on $X$. Further, $T$ makes $F(X)$ into a Hausdorff topological group. The free topology on $F(X)$ is stronger than $T$ and, in general, the topologies are not the same.

3. A fundamental lemma on the topology of $F(X)$. This section is devoted to an important lemma which will be used later in the paper. Before giving this lemma we state another lemma which is a consequence of general topology. 
LEMMA 1. Let $x_{1}, x_{2}, \ldots, x_{n}$ be distinct points of a completely regular space $X$. Let $U_{i}$ be a neighborhood of $x_{i}$ in $X$ for $i=1,2, \ldots, n$. Suppose that $U_{i} \cap U_{j}=\varnothing$ whenever $i \neq j$. Then there is a continuous pseudo-metric $\rho$ on $X$ such that $\rho\left(x, x_{i}\right)=1$ whenever $x \notin U_{i}$ for $i=1,2, \ldots, n$.

Fundamental Lemma. Let $X$ be a completely regular space. Let $x_{1}^{\epsilon_{1}} x_{2}^{\epsilon}$ $\cdots x_{n}^{\epsilon_{n}}$ be a point of $F_{n}(X)$ where $x_{i} \in X, x_{i} \neq e$, and $\epsilon_{i}$ is 1 or -1 for $i=1$, $2, \ldots, n$; and $x_{j} \neq x_{j+1}$ wherever $\epsilon_{j}=-\epsilon_{j+1}$ for $j=1,2, \ldots, n-1$. That is, the word $x_{1}^{\epsilon} x_{2}^{\epsilon} \cdots x_{n}^{\epsilon_{n}}$ is in reduced form. Then a base for the neighborhood system of $x_{1}^{\epsilon_{1}} x_{2}^{\epsilon_{2}} \cdots x_{n}^{\epsilon_{n}}$ in the subspace $F_{n}(X)$ is formed by the family of all sets of the form $U_{1}^{\epsilon_{1}} U_{2}^{\epsilon_{2}} \cdots U_{n}^{\epsilon_{n}}$ where $U_{i}$ is a neighborhood of $x_{i}$ in $X$ for $i=1,2, \ldots, n$.

Proof. Let $V$ be a neighborhood of $x_{1}^{\epsilon} x_{2}^{\epsilon} \cdots x_{n}^{\epsilon_{n}}$ in $F(X)$ for the free topology. By continuity of multiplication we can find neighborhoods $U_{1}^{\prime}, U_{2}^{\prime}$, $\ldots, U_{n}^{\prime}$ of $x_{1}^{\epsilon_{1}}, x_{2}^{\epsilon_{2}}, \ldots, x_{n}^{\epsilon_{n}}$ respectively in $F(X)$ such that $U_{1}^{\prime} U_{2}^{\prime} \cdots U_{n}^{\prime} \subset V$. Define $U_{i}=U_{i}^{\prime} \epsilon_{i} \cap X$ for $i=1,2, \ldots, n$ where $X$ is regarded as a subspace of $F(X)$. Then we have $U_{1}^{\epsilon_{1}} U_{2}^{\epsilon_{2}} \cdots U_{n}^{\epsilon_{n}} \subset V \cap F_{n}(X)$, and $U_{i}$ is a neighborhood of $x_{i}$ in $X$ for $i=1,2, \ldots, n$. It follows that every neighborhood of $x_{1}^{\epsilon_{1}} x_{2}^{\epsilon_{2}}$ $\cdots x_{n}^{\epsilon_{n}}$ in the subspace $F_{n}(X)$ contains a set of the form $U_{1}^{\epsilon_{1}} U_{2}^{\epsilon_{2}} \cdots U_{n}^{\epsilon_{n}}$ where $U_{i}$ is a neighborhood of $x_{i}$ in $X$ for $i=1,2, \ldots, n$.

Let $U_{i}$ be a neighborhood of $x_{i}$ in $X$ for $i=1,2, \ldots, n$. We also require that the sets $U_{i}$ satisfy conditions (i) $e \notin U_{i}$; (ii) $U_{i} \cap U_{j}=\varnothing$ whenever $x_{i} \neq$ $x_{j}$; and (iii) $U_{i}=U_{j}$ whenever $x_{i}=x_{j}$ for all $i, j=1,2, \ldots, n$. It is sufficient to show that $U_{1}^{\epsilon} U_{2}^{\epsilon_{2}} \cdots U_{n}^{\epsilon_{n}}$ is a neighborhood of $x_{1}^{\epsilon_{1}} x_{2}^{\epsilon} \cdots x_{n}^{\epsilon_{n}}$ in the subspace $F_{n}(X)$. Notice that every reduced word in $U_{1}^{\epsilon_{1}} U_{2}^{\epsilon_{2}} \cdots U_{n}^{\epsilon_{n}}$ has length exactly $n$.

By applying Lemma 1 we define a pseudo-metric $\rho$ on $X$ such that $\rho\left(x_{i}, x\right)$ $=1$ whenever $x \in X \backslash U_{i}$ for $i=1,2, \ldots, n$. We observe that $\rho\left(x_{i}, x_{j}\right)=1$ whenever $x_{i} \neq x_{j}$, and $\rho\left(x_{i}, e\right)=1$ for $i=1,2, \ldots, n$. We extend this pseudometric $\rho$ to the entire free topological group $F(X)$ in the usual way, that is in the way which was outlined previously. We also call the extended pseudo-metric $\rho$, and recall that it induces a topology on $F(X)$ which is weaker than the free topology.

Let $U_{i}^{\prime} \subset F(X)$ be defined for $i=1,2, \ldots, n$ by

$$
U_{i}^{\prime}=\left\{x \in F(X): \rho\left(x, x_{i}\right)<1 / n\right\} .
$$

We will show that

$$
U_{1}^{\prime} \epsilon_{1} U_{2}^{\prime \epsilon_{2}} \cdots U_{n}^{\prime} \epsilon_{n} \cap F_{n}(x) \subset U_{1}^{\epsilon_{1}} U_{2}^{\epsilon_{2}} \cdots U_{n}^{\epsilon_{n}}
$$


so that $U_{1}^{\epsilon_{1}} U_{2}^{\epsilon_{2}} \cdots U_{n}^{\epsilon_{n}}$ is a neighborhood of $x_{1}^{\epsilon_{1}} x_{2}^{\epsilon_{2}} \cdots x_{n}^{\epsilon_{n}}$ in $F_{n}(X)$. Notice that $e \notin U_{i}^{\prime}$ for $i=1,2, \ldots, n$.

Let $y_{1} y_{2} \cdots y_{r}$ and $y_{1}^{\prime} y_{2}^{\prime} \cdots y_{r}^{\prime}$ be any two words in $F(X)$ of equal length $r$ with $y_{i}$ and $y_{i}^{\prime}$ in $F_{1}(X)$ for $i=1,2, \ldots, r$ but not necessarily in reduced form. We define

$$
\varphi\left(y_{1} y_{2} \cdots y_{r}, y_{1}^{\prime} y_{2}^{\prime} \cdots y_{r}^{\prime}\right)=\sum_{i=1}^{r} \rho\left(y_{i}, y_{i}^{\prime}\right) .
$$

Notice that $\varphi$ may take on different values for different representations of the same elements.

Let $w_{i}=y_{i 1} y_{i 2} \cdots y_{i k_{i}}$ be a point of $U_{i}^{\prime \epsilon_{i}}$ for $i=1,2, \ldots, n$. We assume further that $y_{i 1} y_{i 2} \cdots y_{i k_{i}}$ is a representation of $w_{i}$ which achieves the distance to $x_{i}^{\epsilon_{i}}=x_{i 1} x_{i 2} \cdots x_{i k_{i}}$. That is,

$$
\rho\left(x_{i}^{\epsilon_{i}}, w_{i}\right)=\sum_{j=1}^{k_{i}} \rho\left(x_{i j}, y_{i j}\right)=\varphi\left(x_{i 1} x_{i 2} \cdots x_{i k_{i}} y_{i 1} y_{i 2} \cdots y_{i k_{i}}\right)
$$

for $i=1,2, \ldots, n$ where the points $x_{i j}$ and $y_{i j}$ are in $F_{1}(X)$ for $i=1,2, \ldots$, $n$ and $j=1,2, \ldots, k_{i}$. Suppose further that

$$
y_{1} \cdots y_{n^{\prime}}=w_{1} \cdots w_{n}=y_{11} \cdots y_{1 k_{1}} \cdots y_{n 1} \cdots y_{n k_{n}}
$$

is a point of $U_{1}^{\prime} \epsilon_{1} U_{2}^{\prime} \epsilon_{2} \cdots U_{n}^{\prime} \epsilon_{n} \cap F_{n}(X)$ where $y_{i}$ is a point of $F_{1}(X)$ for $i=$ $1,2, \ldots, n^{\prime}$. Finally we assume $y_{1} y_{2} \cdots y_{n}^{\prime}$ is in reduced form. We wish to show $n^{\prime}=n$ and $y_{i} \in U_{i}^{\epsilon_{i}}$ for $i=1,2, \ldots, n$. Since $y_{1} y_{2} \cdots y_{n^{\prime}} \in F_{n}(X)$ we know that $n^{\prime} \leqslant n$.

We calculate

$$
\begin{aligned}
& \rho\left(x_{1}^{\epsilon_{1}} \cdots x_{n}^{\epsilon_{n}}, y_{1} \cdots y_{n^{\prime}}\right)=\rho\left(x_{1}^{\epsilon_{1}} \cdots x_{n}^{\epsilon_{n}}, w_{1} \cdots w_{n}\right) \\
& \quad \leqslant \varphi\left(x_{11} \cdots x_{1 k_{1}} \cdots x_{n 1} \cdots x_{n k_{n}}, y_{11} \cdots y_{1 k_{1}} \cdots y_{n 1} \cdots y_{n k_{n}}\right) \\
& \quad=\varphi\left(x_{11} \cdots x_{1 k_{1}}, y_{11} \cdots y_{1 k_{1}}\right)+\cdots+\varphi\left(x_{n 1} \cdots x_{n k_{n}}, y_{n 1} \cdots y_{n k_{n}}\right) \\
& \quad=\rho\left(x_{1}^{\epsilon_{1}}, w_{1}\right)+\cdots+\rho\left(x_{n}^{\epsilon_{n}}, w_{n}\right)<\frac{1}{n}+\frac{1}{n}+\cdots+\frac{1}{n}=1 .
\end{aligned}
$$

Thus we have shown

$$
\rho\left(x_{1}^{\epsilon} x_{2}^{\epsilon} \cdots x_{n}^{\epsilon_{n}}, y_{1} y_{2} \cdots y_{n^{\prime}}\right)<1 .
$$

Suppose $x_{1}^{\prime} x_{2}^{\prime} \cdots x_{k}^{\prime}=x_{1}^{\epsilon} x_{2}^{\epsilon} \cdots x_{n}^{\epsilon_{n}}$ and $y_{1}^{\prime} y_{2}^{\prime} \cdots y_{k}^{\prime}=y_{1} y_{2} \cdots y_{n^{\prime}}$ are words with $x_{i}^{\prime}$ and $y_{i}^{\prime}$ in $F_{1}(X)$ for $i=1,2, \ldots, k$ which realize the distance $\rho\left(x_{1}^{\epsilon} x_{2}^{\epsilon} \cdots x_{n}^{\epsilon_{n}}, y_{1} y_{2} \cdots y_{n^{\prime}}\right)$. That is,

$$
\rho\left(x_{1}^{\epsilon_{1}} x_{2}^{\epsilon} \cdots x_{n}^{\epsilon_{n}}, y_{1} y_{2} \cdots y_{n^{\prime}}\right)=\sum_{i=1}^{k} \rho\left(x_{i}^{\prime}, y_{i}^{\prime}\right) \text {. }
$$


Consider the arrangement

$$
\begin{aligned}
& x_{1}^{\prime} x_{2}^{\prime} \cdots x_{k}^{\prime} \\
& y_{1}^{\prime} y_{2}^{\prime} \cdots y_{k}^{\prime} .
\end{aligned}
$$

Let us fix orders of cancellation for reducing the words $x_{1}^{\prime} x_{2}^{\prime} \cdots x_{k}^{\prime}$ and $y_{1}^{\prime} y_{2}^{\prime}$ $\cdots y_{k}^{\prime}$ to their reduced forms $x_{1}^{\epsilon} x_{2}^{\epsilon} \cdots x_{n}^{\epsilon}$ and $y_{1} y_{2} \cdots y_{n^{\prime}}$ respectively. Whenever we cancel elements in what follows we have these orders of cancellation in mind.

Suppose we consider an $x_{i}^{\epsilon_{i}}$ in the top line of (2) which remains when the top line is reduced to its reduced form. Under this $x_{i}^{\epsilon_{i}}$ is an element $u_{i 1}$. If $u_{i 1}$ is an $e$ or one of the $y_{j}$ that remain when the bottom line is reduced to reduced form, then we stop. Otherwise $u_{i 1}$ is cancelled by a $u_{i 1}^{-1}$ occurring somewhere on the bottom line of (2). Above $u_{i 1}^{-1}$ is an element $u_{i 2}$ in the top line. If $u_{i 2}$ is either $e$ or one of the $x_{j}^{\epsilon_{j}}$ that remain when the top line is reduced, then we stop. We continue this process until it stops as it must, since (2) is a finite arrangement. There are four possible cases.

Case 1. The process ends with an $e$ in the bottom line of (2).

Case 2. The process ends with an $e$ in the top line.

Case 3. The process ends with an element $y_{j_{i}}$ on the bottom line which remains when the bottom line is reduced.

Case 4. The process ends with an element $x_{j_{i}}^{\epsilon_{i}}$ on the top line which remains when the top line is reduced.

We wish to show that with our particular words only Case 3 can occur. Then we will show that the process beginning with $x_{i}^{\epsilon_{i}}$ ends with $y_{i}$ for $i=1,2$, $\ldots, n$. This will imply that $n=n^{\prime}$.

The reader may show that neither of the first two cases can occur. Suppose Case 4 occurs for $x_{i}^{\epsilon_{i}}$. Then the total number of $u_{i j}$ generated is odd, say $2 m_{i}$ -1. The process thus ends with $x_{j_{i}}^{\epsilon_{j}}$ occurring over $u_{i, 2 m_{i}-1}$ in the arrangement (2). We calculate

$$
\begin{aligned}
\rho\left(x_{1}^{\epsilon} x_{2}^{\epsilon} \cdots x_{n}^{\epsilon_{n}}, y_{1} y_{2} \cdots y_{n^{\prime}}\right) & \\
& \geqslant \rho\left(x_{i}^{\epsilon_{i}}, u_{i 1}\right)+\rho\left(u_{i 1}^{-1}, u_{i 2}\right)+\rho\left(u_{i 2}^{-1}, u_{i 3}\right)+\cdots+\rho\left(u_{i, 2 m_{i}-1}^{-1}, x_{j_{i}}^{\epsilon_{i}}\right) \\
& =\rho\left(x_{i}^{\epsilon_{i}}, u_{i 1}\right)+\rho\left(u_{i 1}, u_{i 2}^{-1}\right)+\cdots+\rho\left(u_{i, 2 m_{i}-1}, x_{j_{i}}^{-\epsilon_{i}}\right) \geqslant \rho\left(x_{i}^{\epsilon}, x_{j_{i}}^{-\epsilon_{j i}}\right) .
\end{aligned}
$$

The way the pseudo-metric $\rho$ was constructed assures us that $\rho\left(x_{i}^{\epsilon_{i}}, x_{j_{i}}^{-\epsilon_{j_{i}}}\right) \geqslant 1$ if $x_{i}^{\epsilon_{i}} \neq x_{j_{i}}^{-\epsilon_{j i}}$. This contradicts (1). Thus the only way Case 4 can occur is for $x_{i}^{e_{i}}$ to equal $x_{j_{i}}^{-\epsilon_{j i}}$. Since the word $x_{1}^{\epsilon_{1}} x_{2}^{\epsilon_{2}} \cdots x_{n}^{\epsilon_{n}}$ is in reduced form this implies $\left|i-j_{i}\right| \geqslant 2$. There is no loss of generality in assuming that $j_{i} \geqslant i+2$. Thus we 
may assume that $x_{i}^{\epsilon_{i}}$ is to the left of $x_{j_{i}}^{\epsilon_{i}}$ in arrangement (2) and that $x_{i+1}^{\epsilon_{i+1}}$, and possibly more of the $x_{j}^{\epsilon_{j}}$, occurs between $x_{i}^{\epsilon_{i}}$ and $x_{j_{i}}^{\epsilon_{i}}$ in the top line of (2). We will show that Case 4 must also occur for the element $x_{i+1}^{e_{i+1}}$ and that $x_{j_{i+1}}^{\epsilon_{i+1}}$ is also between $x_{i}^{e_{i}}$ and $x_{j_{i}}^{\epsilon_{i}}$. This will lead to a contradiction which implies that Case 4 does not occur at all.

Before showing this we need a definition which is used both now and later in the proof. We say that an element of $F_{1}(X)$ in (2) is in position $p$ or has position number $p$ if it is situated in a position between the columns determined by $u_{i p}$ and $u_{i p}^{-1}$ in arrangement (2). Notice that an element may have many different position numbers or no position numbers at all. This may occur when the $u_{i j}$ do not proceed strictly to the right but instead change directions one or more times while progressing from $x_{i}^{\epsilon_{i}}$ to $x_{j_{i}}^{\epsilon_{i}}$.

Notice also that if an element $y$ lies in the bottom line of (2) and has odd position numbers $p_{1}, p_{2}, \ldots, p_{t}$, and possibly some even position numbers as well, then $y$ must be cancelled by an element $y^{-1}$ having exactly the same odd position numbers $p_{1}, p_{2}, \ldots, p_{t}$. This follows because when $y$ has an odd position number $p$ and $y^{-1}$ is not also in this position, then $y$ lies between $u_{i p}$ and $u_{i p}^{-1}$ on the bottom line and prevents their cancellation. The even position numbers may be different for such a pair $y$ and $y^{-1}$. Similar remarks can be made about the even position numbers of elements occurring in the top line of (2).

Some of the elements $u_{i p}$ and $u_{i p}^{-1}$ may lie outside of the region between $x_{i}^{\epsilon_{i}}$ and $x_{j_{i}}^{\boldsymbol{e}_{j}}$. Thus there may be points that lie to the left of $x_{i}^{\epsilon_{i}}$ or to the right of $x_{i}^{\epsilon_{i}} i_{i}$ in (2) which have position numbers. However, if the sequence of terms $u_{i j}$ and $u_{i j}^{-1}$ does get outside of the region between $x_{i}^{\epsilon_{i}}$ and $x_{i i}^{\epsilon j_{i}}$, then it must return since the net sum of all its wanderings must be movement from $x_{i}^{e_{i}}$ to $x_{i_{i}}^{\epsilon_{i}}$. Thus we see that the total number of position numbers for a point which is not one of the terms $u_{i j}$ or $u_{i j}^{-1}$ and which lies outside of the region between $x_{i}^{e} i$ and $\boldsymbol{x}_{\boldsymbol{j}_{i}}^{\boldsymbol{\epsilon}_{i}}$ must be even. Similarly, the total number of position numbers of an element which is not one of the terms $u_{i j}$ and $u_{i j}^{-1}$ and which lies in the region between $x_{i}^{\epsilon_{i}}$ and $x_{i_{i}}^{\epsilon_{i}}$ is odd.

Consider the element $x_{i+1}^{\epsilon_{i+1}}$ which does not cancel when the top line is reduced. Since $i<i+1<j_{i}$ we know that $x_{i+1}^{e_{i+1}}$ lies between $x_{i}^{\epsilon_{i}}$ and $x_{j i}^{\epsilon_{j}}$ on the top line. Thus we may conclude that the total number of position numbers for $x_{i+1}^{e_{i+1}}$ is odd and that there are no even position numbers. Clearly $u_{i+1,1}$, which lies directly under $x_{i+1}^{\epsilon_{i+1}}$ has the same position numbers as $x_{i+1}^{\epsilon_{i+1}}$. Thus $u_{i+1,1}$ must cancel since it is an element in the bottom line with an odd, and hence positive, number of odd position numbers. It follows that Case 3 does not occur for $x_{i+1}^{\epsilon_{i+1}}$ with $u_{i+1,1}$ being $y_{j+1}$.

We shall study the sequence $u_{i+1,1}, u_{i+1,1}^{-1}, u_{i+1,2}, u_{i+1,2}^{-1}, \ldots$. Notice that if $j$ is odd then $u_{i+1, j}$ and $u_{i+1, j}^{-1}$ are on the bottom line, while if $j$ is even 
they are on the top line. Suppose that above $u_{i+1, j}^{-1}$ the element $x_{j i+1}^{\epsilon_{j i+1}}$ occurs. Then where convenient we will say that $u_{i+1, j+1}$ is equal to $x_{j_{i+1}}^{\epsilon_{j}}$. Similarly if the element below $u_{i+1, t}^{-1}$ is $y_{i_{i+1}}$, then we will say that $u_{i+1, t+1}$ is equal to $y_{j_{i+1}}$. We show that the following two properties are always satisfied.

(a) If $u_{i+1, j}$ is in the region between the positions of $x_{i}^{\epsilon_{i}}$ and $x_{j_{i}}^{\epsilon_{j}}$ then $u_{i+1, j}$ has an odd number of odd position numbers and an even number of even position numbers.

(B) If $u_{i+1, j}$ is not in the region between the positions of $x_{i}^{\epsilon_{i}}$ and $x_{i_{i}}^{\epsilon_{j}}$ then $u_{i+1, j}$ has an odd number of odd position numbers and also an odd number of even position numbers.

Before giving the proof we notice that whenever $u_{i+1, t}^{-1}$ occurs, the point $u_{i+1, t+1}$ occurs directly above or below it. Thus $u_{i+1, t}^{-1}$ and $u_{i+1, t+1}$ have the same position numbers.

The proof is by induction. We have shown the properties already for $u_{i+1,1}$ which has the same set of position numbers as $x_{i+1}^{\epsilon_{i+1}}$.

Assume the properties are true for $u_{i+1, t}$. We consider the following two cases.

Case (a). Suppose $t$ is odd so that $u_{i+1, t}$ is on the bottom line. Then $u_{i+1, t}^{-1}$ must appear in (2), since by assumption there is an odd, and hence positive, number of odd position numbers. Thus the process cannot stop with $u_{i+1, t}$ being one of the elements $y_{j}$ remaining when the bottom line is reduced. Since $u_{i+1, t}$ is on the bottom line we know $u_{i+1, t}^{-1}$ has exactly the same odd position numbers as $u_{i+1, t}$. Thus $u_{i+1, t}^{-1}$ and $u_{i+1, t+1}$ both have an odd number of odd position numbers. If $u_{i+1, t}^{-1}$ is between $x_{i}^{\epsilon_{i}}$ and $x_{j_{i}}^{\epsilon_{j}}$, then its total number of position numbers is odd so it and $u_{i+1, t+1}$ have an even number of even position numbers as desired. On the other hand, if $u_{i+1, t}^{-1}$ is not between $x_{i}^{\epsilon_{i}}$ and $x_{j i}^{\epsilon_{j}}$, then the total number of position numbers is even so it and $u_{i+1, t+1}$ both have an odd number of even position numbers. In either event properties $(\alpha)$ and $(\beta)$ are true for Case (a).

Case (b). Suppose $t$ is even so that $u_{i+1, t}$ is on the top line. The proof for Case (b) is omitted.

It follows that properties $(\alpha)$ and $(\beta)$ hold for every $u_{i+1, j}$ and $u_{i+1, j}^{-1}$. Suppose $j$ is odd so that $u_{i+1, j}$ is on the bottom line of (2). Then by properties ( $\left.\alpha\right)$ and $(\beta), u_{i+1, j}$ must cancel since it must have an odd, and hence positive, number of odd position numbers. This implies Case 3 cannot occur for $x_{i+1}^{\epsilon_{i+1}}$. Suppose now that $j$ is even and $u_{i+1, j}$ is outside of the region between $x_{i}^{\epsilon_{i}}$ and $x_{j_{i}}^{\epsilon_{i}}$. From property $(\beta)$ we see that it must have an odd, and hence positive, number of even position numbers. Thus $u_{i+1, j}$ must cancel so that Case 4 cannot occur for $x_{i+1}^{\epsilon_{i+1}}$ with such a $u_{i+1, j}$ being $x_{j_{i+1}}^{\epsilon_{j+1}}$. But arrangement (2) is finite so the 
process must end somewhere. The only possibility is for it to end with $x_{j_{i+1}}^{\epsilon_{i+1}}$ in the region between $x_{i}^{\boldsymbol{\epsilon}_{i}}$ and $\boldsymbol{x}_{\boldsymbol{j}_{i}}^{\boldsymbol{\epsilon}_{\boldsymbol{i}}}$.

We have shown that if Case 4 occurs for an element $x_{i}^{\epsilon_{i}}$ with $i<j_{i}$, then Case 4 also occurs for $x_{i+1}$ and $i<j_{i+1}<j_{i}$. Evidently $i<i+1<j_{i+1}<j_{i}$ and $j_{i+1} \geqslant(i+1)+2=i+3$. By applying the same argument successively to $x_{i+1}^{\epsilon_{i+1}}, x_{i+2}^{\epsilon_{i+2}}, \ldots$ we find that Case 4 must occur for all the points $x_{i+1}^{\epsilon_{i+1}}$, $x_{i+2}^{\epsilon_{i+2}}, \ldots$ with $i+t$ being less than $j_{i}$ for $t=1,2, \ldots$. This is impossible so Case 4 cannot occur.

Thus with our particular words in arrangement (2) only Case 3 can occur for any $x_{i}^{\epsilon_{i}}$, for $i=1,2, \ldots, n$, which is not cancelled when the top line is reduced. Since we know $n^{\prime} \leqslant n$, this implies $n=n^{\prime}$.

We still must show that $y_{j_{i}}=y_{i}$ for $i=1,2, \ldots, n$. To do this we will show that $j_{i}<j_{i+1}$ for $i=1,2, \ldots, n-1$. Fix such an $i$. We know that $x_{i+1}^{\epsilon_{i+1}}$ is to the right of $x_{i}^{\epsilon_{i}}$ in (2).

Let us consider the sequence

$$
u_{i 1}, u_{i 1}^{-1}, u_{i 2}, u_{i 2}^{-1}, \ldots, u_{i, 2 m_{i}} u_{i, 2 m_{i}}^{-1} u_{i, 2 m_{i}+1}
$$

where $u_{i, 2 m_{i}+1}=y_{j_{i}}$. This sequence begins at $x_{i}^{\epsilon_{i}}$ and ends at $y_{j_{i}}$. Thus a point which is not a member of this sequence and lies in the region between $x_{i}^{\epsilon_{i}}$ and $y_{i_{i}}$ must have an odd number of position numbers. Points outside of this region must have an even number of position numbers.

We will need the following two properties.

( $\left.\alpha^{\prime}\right)$ If $u_{i+1, j}$ is to the right of $y_{j_{i}}$ then it has an even number of odd position numbers. If $u_{i+1, j}$ is to the left of $y_{j_{i}}$ then it has an odd number of odd position numbers.

( $\left.\beta^{\prime}\right)$ If $u_{i+1, j}$ is to the right of $x_{i}^{\epsilon_{i}}$ then it has an even number of even position numbers. If $u_{i+1, j}$ is to the left of $x_{i}^{\epsilon_{i}}$ then it has an odd number of even position numbers.

Properties $\left(\alpha^{\prime}\right)$ and $\left(\beta^{\prime}\right)$ can be shown by induction by a proof similar to that used to prove $(\alpha)$ and $(\beta)$. This proof is omitted.

Properties $\left(\alpha^{\prime}\right)$ and $\left(\beta^{\prime}\right)$ hold for all points $u_{i+1, j}, j=1,2, \ldots, 2 m_{i+1}$ and also for the point $y_{j_{i+1}}=u_{i+1,2 m_{i+1}+1}$. Now $y_{j_{i+1}}$ is on the bottom line, and it does not cancel when the bottom line is reduced. Thus it does not have any odd position numbers. By $\left(\alpha^{\prime}\right)$ this is impossible if it is to the left of $y_{j_{i}}$. Thus $y_{j_{i+1}}$ is to the right of $y_{i_{i}}$ and $j_{i}<j_{i+1}$ for $i=1,2, \ldots, n-1$ as desired. Since each of the integers $j_{t}$ for $t=1,2, \ldots, n$ must be one of the numbers 1 , $2, \ldots, n$, it follows that $j_{i}=i$ for $i=1,2, \ldots, n$.

Thus we have shown that Case 3 applies to every $x_{i}^{e_{i}}$ which remains when the top line of (2) is reduced. Further we have show that $j_{i}=i$ for 
$i=1,2, \ldots, n$. Going back to Case 3 we calculate for each $i=1,2, \ldots, n$,

$$
\begin{aligned}
& \rho\left(x_{1}^{\epsilon_{1}} x_{2}^{\epsilon_{2}} \cdots x_{n}^{\epsilon_{n}}, y_{1} y_{2} \cdots y_{n}\right) \\
& \geqslant \rho\left(x_{i}^{\epsilon_{i}}, u_{i 1}\right)+\rho\left(u_{i 1}^{-1}, u_{i 2}\right)+\rho\left(u_{i 2}^{-1}, u_{i 3}\right)+\cdots+\rho\left(u_{i, 2}^{-1}, y_{i_{i}}\right) \\
&=\rho\left(x_{i}^{\epsilon_{i}}, u_{i 1}\right)+\rho\left(u_{i 1}, u_{i 2}^{-1}\right)+\rho\left(u_{i 2}^{-1}, u_{i 3}\right)+\cdots+\rho\left(u_{i, 2}^{-1}, y_{i_{i}}\right) \\
& \geqslant \rho\left(x_{i}^{\epsilon_{i}}, y_{i_{i}}\right)=\rho\left(x_{i}^{\epsilon_{i}}, y_{i}\right) .
\end{aligned}
$$

Thus by (1) we have $\rho\left(x_{i}^{\epsilon_{i}}, y_{i}\right)<1$ for $i=1,2, \ldots, n$. Thus $y_{i} \in U_{i}^{\epsilon_{i}}$ for $i=1,2, \ldots, n$. That is,

$$
U_{1}^{\prime} \epsilon_{1} U_{2}^{\prime} \epsilon_{2} \cdots U_{n}^{\prime} \epsilon_{n} \cap F_{n}(X) \subset U_{1}^{\epsilon_{1}} U_{2}^{\epsilon_{2}} \cdots U_{n}^{\epsilon_{n}}
$$

as we set out to show. But $U_{1}^{\prime} \epsilon_{1} U_{2}^{\prime} \epsilon_{2} \cdots U_{n}^{\prime} \epsilon_{n} \cap F_{n}(X)$ is a neighborhood of $x_{1}^{\epsilon_{1}} x_{2}^{\epsilon_{2}} \cdots x_{n}^{\epsilon_{n}}$ in $F_{n}(X)$ so the lemma follows.

A similar proof gives the Abelian case of the Fundamental Lemma.

The following corollary of the Fundamental Lemma is sometimes useful. This result has been announced by Arhangel'skii [3] .

COROLlary 1. Let $X$ be a completely regular space. Then $F_{n}(X)$ and $A_{n}(X)$ are closed subsets of $F(X)$ and $A(X)$ respectively.

PRoof. The corollary follows easily from the Fundamental Lemma. We give an independent proof. Let $\beta(X)$ be the Stone-Čech compactification of $X$. Then there exist a natural continuous monomorphism $\Phi: F(X) \rightarrow F(\beta(X))$ such that $\Phi^{-1}\left(F_{n}(\beta(X))\right)=F_{n}(X)$. Since $\beta(X)$ is compact $F_{n}(\beta(X))$ is compact and hence closed in $F(\beta(X))$. Since $\Phi$ is continuous $F_{n}(X)$ is closed in $F(X)$.

4. Determination of $\operatorname{dim} X$ by $F(X)$ or $A(X)$. Before determining the dimension of $X$ we need the following result.

THEOREM 1. Let $X$ be a completely regular space and let $Y$ be a compact subset of $A(X)$. If the elements of $Y$ are written in terms of the elements of $X$ then the lengths of their reduced representations are bounded. That is $Y$ is contained in $A_{k}(X)$ for some positive integer $k$.

Proof. Suppose $Y$ is not contained in $A_{k}(X)$ for $k=1,2, \ldots$ Then we may choose a sequence $\left\{y_{i}\right\}_{i \geqslant 1}$ contained in $Y$ such that the length of $y_{k}$ in terms of $X$ is at least $2^{k}+\sum_{i=1}^{k-1} n_{i}$ for $k=1,2, \ldots$, where $n_{i}$ denotes the length of the reduced representation of $y_{i}$ with respect to $X$.

Since $Y$ is compact we know that the sequence $\left\{y_{i}\right\}_{i \geqslant 1}$ has at least one limit point $y \in Y$. Let $\epsilon_{1} x_{1}+\epsilon_{2} x_{2}+\cdots+\epsilon_{n} x_{n}=y$ be the reduced representation of $y$ in terms of elements $x_{i}$ of $X$ where $\epsilon_{i}$ is 1 or -1 for $i=1,2, \ldots, n$. 
Similarly let $\epsilon_{i 1} x_{i 1}+\epsilon_{i 2} x_{i 2}+\cdots+\epsilon_{i n_{i}} x_{i n_{i}}=y_{i}$ be the reduced representation of $y_{i}$ for $i=1,2, \ldots$, in terms of $X$ where $\epsilon_{i j}$ is 1 or -1 for $j=1,2, \ldots, n_{i}$.

By adopting an idea used in the proof of Lemma 6.1 [6], we will define inductively continuous functions $\left\{f_{i}\right\}_{i \geqslant 1}$ from $X$ into the additive real numbers. We define $f_{1}$ on $X$ so that $f_{1}(0)=0$ and $f_{1}\left(x_{j}\right)=0$ for $j=1,2, \ldots, n ; f_{1}\left(x_{1 j}\right)$ $=\epsilon_{1 j} 2^{-1}$ for $j=1,2, \ldots, n_{1}$ whenever $x_{1 j} \neq x_{i}$ for $i=1,2, \ldots, n$; and $\left|f_{1}(x)\right| \leqslant 2^{-1}$ for all $x \in X$. Since $X$ is completely regular, such a continuous function exists. Suppose the functions $f_{1}, f_{2}, \ldots, f_{k-1}$ have been chosen. Then define the function $f_{k}$ on $X$ so that $f_{k}(0)=0$ and $f_{k}\left(x_{j}\right)=0$ for $j=1,2, \ldots$, $n ; f_{k}\left(x_{i j}\right)=0$ for $i=1,2, \ldots, k-1$ and $j=1,2, \ldots, n_{i} ;\left|f_{k}(x)\right| \leqslant 2^{-k}$ for all $x \in X$; and $f_{k}\left(x_{k j}\right)=\alpha_{k} \epsilon_{k j} 2^{-k}$ whenever $x_{k j} \neq x_{i}$ for $i=1,2, \ldots, n$ and $x_{k j} \neq x_{i t}$ for $i=1,2, \ldots, k-1$ and $t=1,2, \ldots, n_{i}$ and where $\alpha_{k}$ is 1 or -1 depending on whether $\Sigma_{i=1}^{k-1} \Sigma_{j=1}^{n} \epsilon_{k j} f_{i}\left(x_{k j}\right)$ is nonnegative or negative.

Define the function $f^{\prime}$ from $X$ into the additive group of real numbers by $f^{\prime}(x)=\sum_{i=1}^{\infty} f_{i}(x)$ for all $x \in X$. The function $f^{\prime}$ is continuous on $X$. Extend $f^{\prime}$ to a continuous homomorphism $f$ defined on all of $A(X)$ by

$$
f\left(\epsilon_{1}^{\prime} z_{1}+\epsilon_{2}^{\prime} z_{2}+\cdots+\epsilon_{t}^{\prime} z_{t}\right)=\sum_{i=1}^{t} \epsilon_{i}^{\prime} f^{\prime}\left(z_{i}\right)
$$

where $z_{i} \in X$ and $\epsilon_{i}^{\prime}$ is 1 or -1 for $i=1,2, \ldots, t$. We have for any $k=1$, $2, \ldots$,

$$
\begin{aligned}
\left|f\left(y_{k}\right)\right| & =\left|f\left(\epsilon_{k 1} x_{k 1}+\epsilon_{k 2} x_{k 2}+\cdots+\epsilon_{k n_{k}} x_{k n_{k}}\right)\right|=\left|\sum_{j=1}^{n_{k}} \epsilon_{k j} f^{\prime}\left(x_{k j}\right)\right| \\
& =\left|\sum_{j=1}^{n_{k}} \sum_{i=1}^{\infty} \epsilon_{k j} f_{i}\left(x_{k j}\right)\right|=\left|\sum_{j=1}^{n_{k}} \sum_{i=1}^{k} \epsilon_{k j} f_{i}\left(x_{k j}\right)\right| \\
& =\left|\sum_{i=1}^{k-1} \sum_{j=1}^{n_{k}} \epsilon_{k j} f_{i}\left(x_{k j}\right)+\sum_{j=1}^{n_{k}} \epsilon_{k j} f_{k}\left(x_{k j}\right)\right| .
\end{aligned}
$$

By using the definition of $\alpha_{k}$ we find that the two sums inside the last absolute value have the same sign. Thus we have

$$
\left|f\left(y_{k}\right)\right| \geqslant\left|\sum_{j=1}^{n_{k}} \epsilon_{k j} f_{k}\left(x_{k j}\right)\right| .
$$

The way the functions $f_{k}$ were chosen assures us that every nonzero term in the last sum has the same sign and magnitude $2^{-k}$. Since $n_{k} \geqslant 2^{k}+\sum_{i=1}^{k-1} n_{i}$ we know that at least $n_{k}-\left(\sum_{i=1}^{k-1} n_{i}+n\right) \geqslant 2^{k}-n$ of these terms are not zero. Thus for any $k$ large enough so that $2^{k}-n>2^{k-1}$ or $2^{k-1}>n$ we have $f\left(y_{k}\right) \mid$ $>1 / 2$. But 


$$
f(y)=f\left(\epsilon_{1} x_{1}+\epsilon_{2} x_{2}+\cdots+\epsilon_{n} x_{n}\right)=\sum_{i=1}^{n} \epsilon_{i} f^{\prime}\left(x_{i}\right)=0
$$

so $y$ cannot be a limit point of $\left\{y_{k}\right\}_{k \geqslant 1}$. This is a contradiction. The theorem follows.

Theorem 1 is not a new result. There is a much easier proof which establishes the theorem in both the Abelian and non-Abelian cases. Let $X$ be a completely regular space and $\beta(X)$ its Stone-Cech compactification. Then there is a natural continuous monomorphism $\Phi: F(X) \rightarrow F(\beta(X))$. Let $Y$ be a compact subset of $F(X)$. Then $\Phi(Y)$ is a compact subset of $F(\beta(X))$. By Lemma 9.3 of Steenrod [19] and Theorem 4 of Graev [6] of $F(\beta(X))$ is contained in $F_{n}(\beta(X))$ for some $n$. Thus $Y \subset \Phi^{-1}\left(F_{n}(\beta(X))\right)=F_{n}(X)$.

The first proof is given because it does not use the fact that $A(X)$ is a free Abelian topological group on $X$. All that is used is that every continuous map from $X$ into the additive group of real numbers $R$ can be extended to a continuous homomorphism from $A(X)$ into $R$. This is a much weaker condition which is satisfied by $F(X, \underline{\underline{V}})$, the free topological group on $X$ of $\underline{\underline{V}}$, where $\underline{\underline{V}}$ denotes any Abelian variety of topological groups containing $R$. For information on varieties of topological groups see [12], [13], and [14] by S. A. Morris.

Let us recall a few facts about dimension [15]. The empty set is said to have weak inductive dimension -1. A topological space $X$ is said to have weak inductive dimension $\leqslant n$ if every point $x$ in $X$ has a fundamental system of neighborhoods whose boundaries have weak inductive dimension $\leqslant n-1$. We say the weak inductive dimension of $X$ is equal to $n$ if it is $\leqslant n$ but not $\leqslant n-1$. Weak inductive dimension is equivalent to the other standard definitions of dimension for a separable metric space.

In Graev [6] it is shown that if $X$ and $Y$ are compact metric spaces such that $A(X)$ and $A(Y)$ are topologically isomorphic then $X$ and $Y$ have the same dimension. By combining some ideas of Graev's proof with some of our own we are able to extend this result to the case where $X$ and $Y$ are locally compact metric spaces. The results also hold if $F(X)$ and $F(Y)$ are topologically isomorphic.

THEOREM 2. Let $X$ and $Y$ be locally compact metric spaces with $A(X)$ topologically isomorphic to $A(Y)$. Then the spaces $X$ and $Y$ have the same weak inductive dimension.

Proof. We will regard $Y$ as a subset of $A(X)$ which generates $A(X)$ as its free topological group. In this way we can write elements of $X$ in terms of elements of $Y$ and elements of $Y$ in terms of elements of $X$. 
Let $\eta_{k}(X)$ be the set of all points $x$ in $X$ where the dimension of $X$ is at least $k$; that is, where $\operatorname{dim}_{x} X \geqslant k$. Suppose $\operatorname{dim} X \geqslant k$ so that $\eta_{k}(X)$ is not empty. Then from dimension theory [2, p. 42], we know for $x \in \eta_{k}(X)$ that $\operatorname{dim}_{x} \overline{\eta_{k}(X)}=\operatorname{dim}_{x} X \geqslant k$. Let $X_{0}$ be the nonempty compact closure in $\overline{\eta_{k}(X)}$ of an open set. Then $X_{0}$ is a compact subset of $X$ which contains a dense set of points $x$ such that $\operatorname{dim}_{x} X_{0} \geqslant k$. It follows that the dimension of every open subset of $X_{0}$ is at least $k$.

Since $X_{0}$ is compact we know from Theorem 1 that the points of $X_{0}$ can be written as words of bounded length with respect to $Y$. Let $n$ be the smallest positive integer for which $X_{0} \subset A_{n}(Y)$. Let $x \in X_{0}$ have reduced representation $y_{1}+y_{2}+\cdots+y_{n}$ where $y_{i} \in A_{1}(Y)$ for $i=1,2, \ldots, n$ and where the set $S=\left\{(i, j): y_{i}=y_{j}\right.$ and $\left.j \neq i\right\}$ contain a minimum number of pairs $(i, j)$. Choose open sets $U_{1}, U_{2}, \ldots, U_{n}$ with compact closures in $A_{1}(Y)$ so that $0 \notin U_{i}$, either $U_{i} \subset Y$ on $U_{i} \subset Y^{-1}$, and $y_{i} \in U_{i}$ for $i=1,2, \ldots, n ; U_{i} \cap U_{j}^{-1}=\varnothing$ for $i, j=1,2, \ldots, n ; U_{i} \cap U_{j}=\varnothing$ whenever $(i, j) \notin S$; and $U_{i}=U_{j}$ whenever $(i, j) \in S$. Define

$$
V=\left(U_{1}+U_{2}+\cdots+U_{n}\right) \cap X_{0} .
$$

Then by the Fundamental Lemma, $V$ is an open neighborhood of $x$ in $X_{0}$ such that every element of $V$ has length exactly $n$ with respect to $Y$. Further, if $\left(y_{1}^{\prime}+y_{2}^{\prime}+\cdots+y_{n}^{\prime}\right) \in V$ then $y_{i}^{\prime}=y_{j}^{\prime}$ if and only if $(i, j) \in S$.

Every point $p \in V$ can be written uniquely in the form $p_{1}+p_{2}+\cdots+$ $p_{n}$ where $p_{i} \in U_{i}$ for $i=1,2, \ldots, n$. Define functions $f_{i}$ from $V$ into $U_{i}$ by $f_{i}(p)=p_{i}$ for $i=1,2, \ldots, n$ and for $p=p_{1}+p_{2}+\cdots+p_{n} \in V$. We will show that the functions $f_{i}$ are continuous on $V$. Suppose $p=p_{1}+p_{2}+\cdots+$ $p_{n} \in V$ and $W_{i}$ is an open neighborhood of $f_{i}(p)=p_{i}$ in $A_{1}(Y)$. We may assume without loss of generality that $W_{i} \subset U_{i}$ for $i=1,2, \ldots, n$. We have

$$
\begin{aligned}
f^{-1}\left(W_{i}\right) & =\left\{p_{1}^{\prime}+p_{2}^{\prime}+\cdots+p_{n}^{\prime} \in V: p_{i}^{\prime} \in W_{i}\right\} \\
& =\left(U_{1}+U_{2}+\cdots+U_{i-1}+W_{i}+U_{i+1}+\cdots+U_{n}\right) \cap V .
\end{aligned}
$$

It follows that $f_{i}^{-1}\left(W_{i}\right)$ is a neighborhood of $p$ in $V$ so that $f_{i}$ is continuous in $V$ for $i=1,2, \ldots, n$.

First we consider the function $f_{1}$. Let $y_{1}^{\prime}=x_{11}+x_{12}+\cdots+x_{1 n_{1}}$ be a point of maximal length $n_{1}$ in $f_{1}(V)$ with respect to $X$. We also require that the set $S_{1}=\left\{(i, j): x_{1 i}=x_{1 j}\right.$ and $\left.i \neq j\right\}$ contain a minimum number of pairs $(i, j)$. Choose open sets $V_{11}, V_{12}, \ldots, V_{1 n_{1}}$ with compact closures in $A_{1}(X)$ so that $0 \notin V_{1 i}, V_{1 i} \subset X$ or $V_{1 i} \subset X^{-1}$, and $x_{1 i} \in V_{1 i}$ for $i=1,2, \ldots, n$; $V_{1 i} \cap V_{1 j}^{-1}=\varnothing$ for $i, j=1,2, \ldots, n_{1} ; V_{1 i} \cap V_{1 j}=\varnothing$ whenever $(i, j) \notin S_{1}$; $V_{1 i}=V_{1 j}$ whenever $(i, j) \in S_{1}$; and $\left(V_{11}+V_{12}+\cdots+V_{1 n_{1}}\right) \cap A_{1}(Y) \subset U_{1}$. 
Define $W_{1}$ to be an open set in $A(X)$ such that $W_{1} \cap A_{n_{1}}(X)=V_{11}+V_{12}$ $+\cdots+V_{1 n_{1}}$ and $W_{1} \cap A_{1}(Y) \subset U_{1}$. Define $U_{1}^{\prime}=W_{1} \cap A_{1}(Y)$. Then $U_{1}^{\prime}$ is an open neighborhood of $y_{1}^{\prime}$ in $A_{1}(Y)$ such that every point of $f_{1}(V) \cap U_{1}^{\prime}$ has length exactly $n_{1}$ with respect to $X$ and $U_{1}^{\prime} \subset U_{1}$. Since $y_{1}^{\prime} \in f_{1}(V)$, there is a point of the form $y_{1}^{\prime}+y_{12}+y_{13}+\cdots+y_{1 n} \in V \subset X_{0}$ with $y_{1 i} \in U_{t}$ for $i=2,3, \ldots, n$. Define

$$
V_{1}=\left(U_{1}^{\prime}+U_{2}+U_{3}+\cdots+U_{n}\right) \cap X_{0} .
$$

Then $y_{1}^{\prime}+y_{12}+y_{13}+\cdots+y_{1 n} \in V_{1} \subset V ; V_{1}$ is open in $X_{0}$; and for any point $z_{1}+z_{2}+\cdots+z_{n} \in V_{1}$ with $z_{i} \in F_{1}(Y)$ for $i=1,2, \ldots, n$, we know $z_{1}$ has length exactly $n_{1}$ with respect to $X$. As $V_{1} \subset V$, we know every element in $V_{1}$ has length exactly $n$ with respect to $Y$.

We are now able to apply the same argument using $f_{2}$ instead of $f_{1}$ and $V_{1}$ instead of $V$. After this we continue by applying this argument successively to $f_{3}, f_{4}, \ldots, f_{n}$. At the end of this process we will have open sets $V_{i j}$ with compact closures in $A_{1}(X)$ for $i=1,2, \ldots, n$ and $j=1,2, \ldots, n_{i}$ so that: $0 \notin$ $V_{i j}$ and either $V_{i j} \subset X$ or $V_{i j} \subset X^{-1}$ for $i=1,2, \ldots, n$ and $j=1,2, \ldots, n_{i}$; $V_{i, j} \cap V_{i, t}^{-1}=\varnothing$ for $i=1,2, \ldots, n$ and $j, t=1,2, \ldots, n_{i} ; V_{i s} \cap V_{i t}=\varnothing$ whenever $(s, t) \notin S_{i} ; V_{i s}=V_{i t}$ whenever $(s, t) \in S_{i}$; and $\left(V_{i 1}+V_{i 2}+\cdots+\right.$ $\left.V_{i n_{i}}\right) \cap A_{1}(Y) \subset U_{i}$. During this process we let $W_{i}$ be an open set in $A(X)$ such that $W_{i} \cap A_{n_{i}}(X)=V_{i 1}+V_{i 2}+\cdots+V_{i n_{i}}$ and $W_{i} \cap A_{1}(Y) \subset U_{i}$ for $i=1$, $2, \ldots, n$. We also let $U_{i}^{\prime}=W_{i} \cap A_{1}(Y)$ and $V_{i}=\left(U_{1}^{\prime}+U_{2}^{\prime}+\cdots+U_{i}^{\prime}+\right.$ $\left.U_{i+1}+\cdots+U_{n}\right) \cap X_{0}$ for $i=1,2, \ldots, n$. Each $U_{i}^{\prime}$ is a nonempty open subset of $A_{1}(Y)$ and each $V_{i}$ is a nonempty open subset of $V \subset X_{0}$. We have $V \supset$ $V_{1} \supset V_{2} \supset \cdots \supset V_{n}$.

It is $V_{n}$ which interests us. Every point in $V_{n}$ has length exactly $n$ with respect to $Y$. If $z_{1}+z_{2}+\cdots+z_{n}$ is any point of $V_{n}$ with each $z_{i} \in U_{i}^{\prime} \subset$ $A_{1}(Y)$ then $z_{i}$ has length exactly $n_{i}$ with respect to $X$ for $i=1,2, \ldots, n$.

Let $z_{i 1}+z_{i 2}+\cdots+z_{i n_{i}}$ be arbitrary points of $U_{i}^{\prime} \cap f_{i}\left(V_{n}\right)$ for $i=1,2$, $\ldots, n$ where $z_{i j} \in V_{i j}$ for $i=1,2, \ldots, n$ and $j=1,2, \ldots, n_{i}$. Define functions $g_{i j}$ from $U_{i}^{\prime} \cap f_{i}\left(V_{n}\right)$ into $V_{i j}$ by $g_{i j}\left(z_{i 1}+z_{i 2}+\cdots+z_{i n_{i}}\right)=z_{i j}$ for $i=$ $1,2, \ldots, n$ and $j=1,2, \ldots, n_{i}$. Since every point of $U_{i}^{\prime} \cap f_{i}\left(V_{n}\right)$ has a unique representation of the form $z_{i 1}+z_{i 2}+\cdots+z_{i n_{i}}$ these functions are well defined. As was the case for the functions $f_{i}$ we know $g_{i j}$ is continuous for $i=$ $1,2, \ldots, n$ and $j=1,2, \ldots, n_{i}$.

We shall study the functions $g_{i j} \circ f_{i}$ from $V_{n} \subset X_{0}$ into $V_{i j} \subset X$. Suppose $x$ is any point of $V_{n}$. Then we have the equation $x=y_{1}+y_{2}+\cdots+y_{n}=$ $x_{11}+\cdots+x_{1 n_{1}}+\cdots+x_{n 1}+\cdots+x_{n n_{n}}$ for suitable choices of $y_{i}=x_{i 1}$ $+x_{i 2}+\cdots+x_{i n_{i}} \in U_{i}^{\prime} \cap f_{1}\left(V_{n}\right)$, where $x_{i j} \in V_{i j}$ for $i=1,2, \ldots, n$ and 
$j=1,2, \ldots, n_{i}$. From this equation we know the word

$$
\begin{aligned}
x_{11}+x_{12} & +\cdots+x_{1 n_{1}}+x_{21}+x_{22} \\
& +\cdots+x_{2 n_{2}}+\cdots+x_{n 1}+x_{n 2}+\cdots+x_{n n_{n}}
\end{aligned}
$$

reduces to simply $x$. It follows that for any $x \in V_{n}$ there are values of $i$ and $j$ for which $g_{i j} \circ f_{i}(x)=x_{i j}=x$. Let $A_{i j}$ be the set of all fixed points of $g_{i j} \circ f_{i}$ in $V_{n}$. Then

$$
V_{n}=\bigcup_{1<i<n ; 1<j<n_{i}} A_{i j}
$$

In addition $A_{i j}$ is closed in $V_{n}$ for $i=1,2, \ldots, n$ and $j=1,2, \ldots, n_{i}$. Since this is a finite union, we can choose integers $s$ and $t$ so that $A_{s t}$ contains a nonempty open set $W_{s t}$ in $V_{n}$. By taking a smaller set if necessary we assume $\bar{W}_{s t}$ $\subset V_{n}$ where the closure is taken in the metric space $X_{0}$.

We know $g_{s t} \circ f_{s}$ is the identity on $\bar{W}_{s t}$. Thus $f_{s}$ is one-to-one on $\bar{W}_{s t}$. We know $f_{s}$ is continuous and $\bar{W}_{s t}$ is compact. It follows that $f_{s} \mid \bar{W}_{s y}$ is a homeomorphism from $\bar{W}_{s t}$ into $U_{s}^{\prime} \subset F_{1}(Y)$. Recall that either $U_{s}^{\prime} \subset Y$ or $U_{s}^{\prime} \subset Y^{-1}$.

Since $Y$ and $Y^{-1}$ are homeomorphic we know that in either case $Y$ contains a copy $f_{s}\left(W_{s t}\right)$ of $W_{s t}$. But $W_{s t}$ is a nonempty open subset of $X_{0}=\overline{\eta_{k}(X)}$ so $\operatorname{dim} W_{s t} \geqslant k$. It follows that $\operatorname{dim} Y \geqslant \operatorname{dim} f_{s}\left(W_{s t}\right) \geqslant k$. Hence $\operatorname{dim} X \leqslant \operatorname{dim} Y$.

In the same way $\operatorname{dim} Y \leqslant \operatorname{dim} X$. Thus $\operatorname{dim} X=\operatorname{dim} Y$ as desired.

THEOREM 3. Let $X$ and $Y$ be locally compact metric spaces with $F(X)$ topologically isomorphic to $F(Y)$. Then the spaces $X$ and $Y$ have the same weak inductive dimension.

Proof. By Markov [10], $A(X)=F(X) / \delta$ and $A(Y)=F(Y) / \delta$ where $\delta$ is the commutator subgroup. Thus $A(X) \cong A(Y)$. The result follows from Theorem 2.

5. Miscellaneous results. Next we take advantage of the theory we have developed to investigate what conditions the space $X$ must satisfy in order for $F(X)$ and $A(X)$ to be locally compact, paracompact, or normal. If $X$ has the discrete topology then $F(X)$ and $A(X)$ also have the discrete topology. Graev showed in $C$ which follows his Lemma 6.1 that if $X$ is not discrete then the topological groups $F(X)$ and $A(X)$ are not second countable. A close reading of his proof reveals that he shows that if $X$ is not discrete then $F(X)$ and $A(X)$ are not first countable and hence not metrizable. The following consequence of Theorem 1 has also been shown by Dudley [4] and Abels [1]

THEOREM 4. Let $X$ be a completely regular space. If the topology of $X$ is not discrete then the groups $F(X)$ and $A(X)$ are not locally compact. 
Proof. Suppose $U$ is a compact neighborhood of the identity element 0 in $A(X)$. By Theorem $1, U \subset A_{n}(X)$ for some positive integer $n$. Let $x_{0}$ be a nonisolated point of $X$. Then $n x_{0}$ is a limit point of $\left\{n x: x \in X, x \neq x_{0}\right\}$. Thus 0 is a limit point of $\left\{n x-n x_{0}: x \in X, x \neq x_{0}\right\}$. Every point $n x-n x_{0}$ with $x \neq x_{0}$ has length $2 n$ so $U$ contains an element of length $2 n$. This is impossible since $U \subset A_{n}(X)$. Thus there are no compact neighborhoods of 0 and $A(X)$ is not locally compact.

We know [6] that the mapping $\varphi$ from $F(X)$ onto $A(X)$ defined by $\varphi\left(x_{1} x_{2} \cdots x_{n}\right)=x_{1}+x_{2}+\cdots+x_{n}$ is both continuous and open. It follows that $F(X)$ is not locally compact either.

It is easy to see that if $X$ is a completely regular space which is a countable union of compact spaces then the groups $F(X)$ and $A(X)$ are also countable unions of compact spaces. Thus $F(X)$ and $A(X)$ are paracompact. However if $X$ is Lindelof and completely regular (i.e. paracompact) then $F(X)$ and $A(X)$ need not be paracompact. Let $X$ be a completely regular space which is not normal. By constructing $F(X)$ and $A(X)$ for such a space, Markov [10] showed that a topological group or an Abelian topological group need not be normal. Our example will also show that the free topological group and free Abelian topological group of a normal space need not be normal.

ExAmple. Let $X$ be the closed unit interval $[0,1]$ with the half open interval topology. That is a base for the topology is formed by all half open intervals of the form $[x, y) . X$ is a normal Lindelof space for which $F(X)$ and $A(X)$ are not normal. We leave the details of the proof to the reader. The proof uses the Fundamental Lemma and is very much like the standard proof used to show the topological product of $X$ with itself is not normal. Additional relevant information can be found in [20].

Let $G$ be a topological group. We denote by $\operatorname{Aut}(G)$ the set of all topological isomorphisms of $G$ onto itself. Then $\operatorname{Aut}(G)$ is itself a group.

THEOREM 5. Let $X$ be a completely regular space. Let $\varphi \in \operatorname{Aut}(F(X))$. Then the subspace $\varphi(X)$ of $F(X)$ algebraically generates all of $F(X)$ as its free topological group.

Proof. There can be no nontrivial algebraic relations in $\varphi(X)$ as $\varphi^{-1} \in$ $\operatorname{Aut}(F(X))$ and such relations are carried back into $X$ by $\varphi^{-1}$. In addition $e \in$ $\varphi(X)$. Thus $\varphi(X)$ algebraically generates a free group $\langle\varphi(X)\rangle \subset F(X)$. Let $x \in X$ with $\varphi^{-1}(x)=x_{1}^{\epsilon_{1}} x_{2}^{\epsilon_{2}} \cdots x_{n}^{\epsilon_{n}}$ where $x_{i} \in X$ and $\epsilon_{i}$ is 1 or -1 for $i=1,2, \ldots$, n. Then $x=\varphi\left(x_{1}^{\epsilon_{1}} x_{2}^{\epsilon} \cdots x_{n}^{\epsilon_{n}}\right)=\varphi\left(x_{1}\right)^{\epsilon} \varphi^{\epsilon}\left(x_{2}\right)^{\epsilon_{2}} \cdots \varphi\left(x_{n}\right)^{\epsilon_{n}}$ so that $x \in$ $\langle\varphi(X)\rangle$ for every $x \in X$. It follows that $\varphi(X)$ generates the entire free group $F(X)$.

Let us show that $F(X)$ is the free topological group of $\varphi(X)$. We verify the 
three properties given in the definition of a free topological group. First we observe that $\varphi(X) \subset F(X)$ and second we recall that $\varphi(X)$ algebraically generates $F(X)$. Now suppose $h^{\prime}$ is any continuous function from $\varphi^{\prime}(X)$ into a topological group $G$ such that $h^{\prime}(e)$ is the identity in $G$. We extend $h^{\prime}$ to a function $h$ defined on all of $F(X)$ by $h\left(\varphi\left(x_{1}\right)^{\epsilon_{1}} \cdots \varphi\left(x_{n}\right)^{\epsilon_{n}}\right)=h^{\prime}\left(\varphi\left(x_{1}\right)\right)^{\epsilon_{1}} \cdots h^{\prime}\left(\varphi\left(x_{n}\right)\right)^{\epsilon_{n}}$ where $x_{1}, x_{2}, \ldots, x_{n}$ are points of $X$ and $\epsilon_{i}$ is 1 or -1 for $i=1,2, \ldots, n$. Notice that since $\langle\varphi(X)\rangle=F(X)$ any word in $F(X)$ can be written in the form $\varphi\left(x_{1}\right)^{\epsilon_{1}} \varphi\left(x_{2}\right)^{\epsilon_{2}} \cdots \varphi\left(x_{n}\right)^{\epsilon_{n}}$.

The extended function $h$ is clearly a homomorphism. We must show that it is continuous. Consider the continuous function $\left(h^{\prime} \circ \varphi\right) \mid X$ from $X$ into the group $G$. We know $F(X)$ is the free topological group of $X$ so the extension $g$ of $\left(h^{\prime} \circ \varphi\right) \mid X$ to $F(X)$ defined by $g\left(x_{1}^{\epsilon_{1}} \cdots x_{n}^{\epsilon_{n}}\right)=\left[(h \circ \varphi \mid X)\left(x_{1}\right)\right]^{\epsilon_{1}} \cdots$ $\left[(h \circ \varphi \mid X)\left(x_{n}\right)\right]^{\epsilon_{n}}$ is continuous. A direct calculation shows that $h=g \circ \varphi^{-1}$. Thus $h$ is continuous and $F(X)$ is the free topological group of $\varphi(X)$.

An analogous argument gives the Abelian case of this theorem.

\section{REFERENCES}

1. H. Abels, Normen auf freien topolegischen Gruppen, Math. Z. 129 (1972), 25-42. MR 47 \#3589.

2. P. Alexandroff, Darstellung der Grundzüge der Urysohnschen Dimensionstheorie, Math. Ann. 98 (1927), $31-63$.

3. A. V. Arhangel'skii, Mappings related to topological groups, Dokl. Akad. Nauk SSSR 181 (1968), 1303-1306 = Soviet Math. Dokl. 9 (1968), 1011-1015. MR 38 \#2237.

4. R. M. Dudley, Continuity of homomorphisms, Duke Math. J. 28 (1961), 587-594. MR 25 \#141.

5. J. Dugundji, Topology, Allyn and Bacon, Boston, Mass., 1966. MR 33 \#1824.

6. M. I. Graev, Free topological groups, Izv. Akad. Nauk SSSR Ser. Mat. 12 (1948), 279-324; English transl., Amer. Math. Soc. Transl. (1) 8 (1962), 305-364. MR 10, 11.

7. E. Hewitt and K. A. Ross, Abstract harmonic analysis. Vol. 1: Structure of topological groups. Integration theory, group representations, Die Grundlehren der math. Wissenschaften, Band 115, Academic Press, New York; Springer-Verlag, Berlin, 1963. MR 28 \#158.

8. S. Kakutani, Free topological groups and infinite direct product topological groups, Proc. Imp. Acad. Tokyo 20 (1944), 595-598. MR 7, 240. 1136.

9. J. L. Kelley, General topology, Van Nostrand, Princeton, N. J., 1955. MR 16,

10. A. A. Markov, On free topological groups, Izv. Akad. Nauk SSSR Ser. Mat. 9 (1945), 3-64; English transl., Amer. Math. Soc. Transl. (1) 8 (1962), 195-272. MR $7,7$.

11. A. S. Miščenko, Dimension of groups with a left-invariant topology, Dokl. Akad. Nauk SSSR 159 (1964), 753-754 = Soviet Math. Dokl. 5 (1964), 1603-1605. MR 30 \#3446.

12. S. A. Morris, Varieties of topological groups. I, Bull. Austral. Math. Soc. 1 (1969), 145-160. MR 41 \#3655a.

13. - Varieties of topological groups. II, Bull. Austral. Math. Soc. 2 (1970), 1-13. MR 41 \#3655b.

14. - Varieties of topological groups. III, Bun. Austral. Math. Soc. 2 (1970), 165-178. MR 41 \#3655c.

15. J. Nagata, Modern dimension theory, Bibliotheca Math., vol. 6, Interscience, New York, 1965. MR 34 \#8380. 
16. T. Nakayama, Note on free topological groups, Proc. Imp. Acad. Tokyo 19 (1943), 471-475. MR 7, 240.

17. L. S. Pontrjagin, Continuous groups, 2nd ed., GITTL, Moscow, 1954; English transl., Gordon and Breach, New York, 1966. MR 17, 171; 34 \#1439.

18. P. Samuel, On universal mappings and free topological groups, Bull. Amer. Math. Soc. 54 (1948), 591-598. MR 9, 605.

19. N. E. Steenrod, A convenient category of topological spaces, Michigan Math. J. 14 (1967), 133-152. MR 35 \#970.

20. B. V. S. Thomas, Free topological groups, General Topology and Appl. 4 (1974), 51-72. MR 49 \#7385.

DEPARTMENT OF MATHEMATICS, CASE WESTERN RESERVE UNIVERSITY, CLEVELAND, OHIO 44106 